\title{
Search for Neutral D Meson Mixing using Semileptonic Decays
}

\author{
Kevin Flood \\ Stanford Linear Accelerator Center \\ Stanford University \\ Stanford, CA 94309
}

SLAC-Report-818

Prepared for the Department of Energy

under contract number DE-AC02-76SF00515

Printed in the United States of America. Available from the National Technical Information Service, U.S. Department of Commerce, 5285 Port Royal Road, Springfield, VA 22161. 
This document, and the material and data contained therein, was developed under sponsorship of the United States Government. Neither the United States nor the Department of Energy, nor the Leland Stanford Junior University, nor their employees, nor their respective contractors, subcontractors, or their employees, makes an warranty, express or implied, or assumes any liability of responsibility for accuracy, completeness or usefulness of any information, apparatus, product or process disclosed, or represents that its use will not infringe privately owned rights. Mention of any product, its manufacturer, or suppliers shall not, nor is it intended to, imply approval, disapproval, or fitness of any particular use. A royalty-free, nonexclusive right to use and disseminate same of whatsoever, is expressly reserved to the United States and the University. 


\title{
SEARCH FOR NEUTRAL $D$ MESON MIXING USING SEMILEPTONIC DECAYS
}

\author{
A Dissertation Presented \\ by \\ KEVIN T. FLOOD
}

Submitted to the Graduate School of the University of Massachusetts Amherst in partial fulfillment of the requirements for the degree of

\section{DOCTOR OF PHILOSOPHY}

May 2004

Department of Physics 
(C) Copyright by Kevin T. Flood 2004

All Rights Reserved 
To all the lost sheep and the good shepherds who search for them. 


\section{ACKNOWLEDGMENTS}

Thanks to my colleagues at UMass and SLAC for their efforts and support. In particular, I would like to thank my UMass advisors, Stan Hertzbach and Guy Blaylock, for their very substantial help and good advice along the way. In addition, I have benefited greatly from many detailed discussions with Mike Sokoloff, a Babar colleague, who took the time to thoroughly understand and critique my analysis, and helped improve it with better particle identification, among other things. I would like to also specifically thank the members of the Babar Charm Physics Analysis Working Group, who have listened to and reviewed my presentations, and given much helpful feedback on both the substance and presentation of my work. It would not have been possible for any Babar analysis to proceed without the hard work and dedication of our PEP-II colleagues, and I am grateful for their commitment to delivering the highest possible luminosity with the lowest possible machine backgrounds. Babar also substantially depends upon the tireless efforts of the computing organizations in several countries that make access to both the data and the resources with which to analyze it possible, and 1 am grateful for the opportunity they have afforded me to use those substantial resources. Ultimately, I would like to express my gratitude to the taxpayers of the many countries collaborating in the B-Factory who made all of this possible in the first place. 


\title{
ABSTRACT \\ SEARCH FOR NEUTRAL $D$ MESON MIXING USING SEMILEPTONIC DECAYS
}

\author{
MAY 2004 \\ KEVIN T. FLOOD \\ B.Sc, UNIVERSITY OF CALIFORNIA SANTA CRUZ \\ M.Sc, UNIVERSITY OF MASSACHUSETTS AMHERST \\ Ph.D., UNIVERSITY OF MASSACHUSETTS AMHERST
}

Directed by: Professor Stanley S, Hertzbach

Based on a $87 \mathrm{fb}^{-1}$ dataset, a search for $D^{0}-\bar{D}^{0}$ mixing is made using the semileptonic decay modes $D^{\alpha+} \rightarrow \pi^{+} D^{0}, D^{0} \rightarrow\left[K / K^{*}\right] e \nu(+c . c$ ) at the $B$-Factory facility at the Stanford Linear Accelerator Center. These modes offer unambiguous initial and final-state charm flavor tags, and allow the combined use of the $D^{0}$ lifetime and $D^{*+}$ $D^{0}$ mass difference $(\Delta M)$ in a global likelihood fit. The high-statistics sample of reconstructed unmixed semileptonic $D^{f}$ decays is used to model both the $\Delta M$ distribution and the time-dependence of mixed events directly from the data. Neural networks are used both to select events and to fully reconstruct the $D^{0}$. A result consistent, with no charm mixing has been obtained, $R_{\operatorname{mix}}=0.0023 \pm 0.0012$ (stat) \pm 0.0004 (sys). This corresponds to an upper limit of $R_{\text {mix }}<0.0047$ (95\% C.L.) and $R_{\text {mir }}<0.0043$ ( $90 \%$ C L.). The lowest current published limit on semileptonic charm mixing is 0.005 (90\% C.L.) (E791, E.M. Aitala et al., Phys.Rev.Lett. 772384 (1996)). The current 
best published limit using any analysis technique on the total rate of charm mixing is 0.0016 (95\% C.L.) (Babar $K \pi$ mixing, B. Aubert et al., Phys.Rev.Lett. 91171801 (2003)). 


\section{TABLE OF CONTENTS}

\section{Page}

ACKNOWLEDGMENTS

$\mathbf{v}$

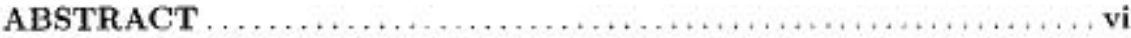

LIST OF TABLES $\ldots \ldots \ldots \ldots \ldots \ldots \ldots \ldots \ldots \ldots \ldots \ldots \ldots \ldots \ldots \ldots \ldots \ldots$

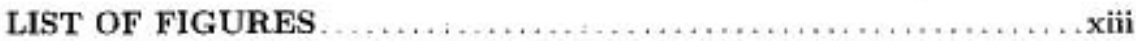

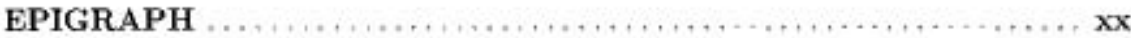

\section{CHAPTER}

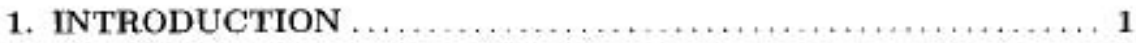

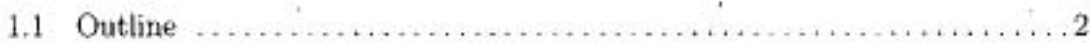

2. THEORY AND PREVIOUS EXPERIMENTAL RESULTS ...... 4

2.1 Charm Mixing Formalism $\ldots, \ldots \ldots \ldots \ldots \ldots \ldots \ldots \ldots \ldots, \ldots, \ldots$

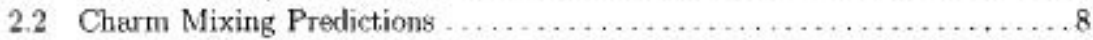

2.2.1 Standard Model Contributions . . . . . . . . . . . . . . . . . . . 8

2.2 .2 New Physics Predictions . . . . . . . . . . . . . . . . . 11

2.3 Previous Experimental Results . . . . . . . . . . . . . . . . . . . . . . 13

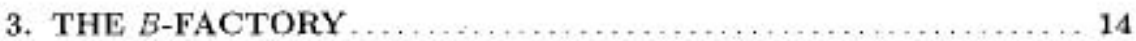

3.1 Data Sample ......................................... 14

3.1.1 Monte Carlo Events .............................. 18

3.2 The Babar Detector ....................................... 19

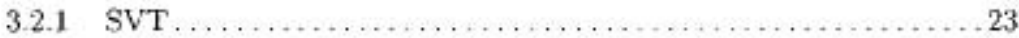




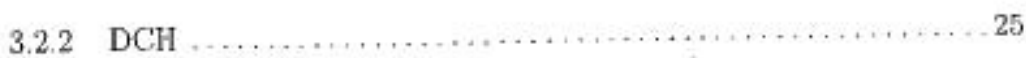

3.2.2.1 Charged Track Reconstruction ................. 32

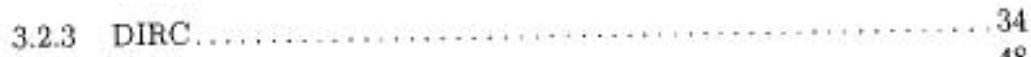

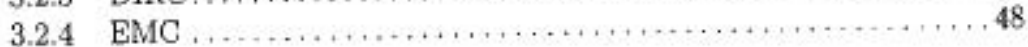

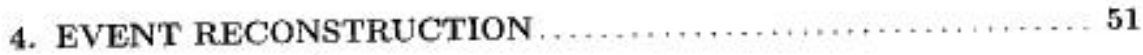

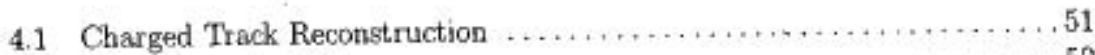

4.2 Pion Reconstruction ............................... 52

4.3 Kaon Reconstruction................................. 53

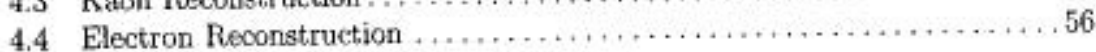

4.4.1 Gamma Conversion Veto .......................67 67

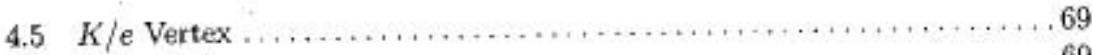

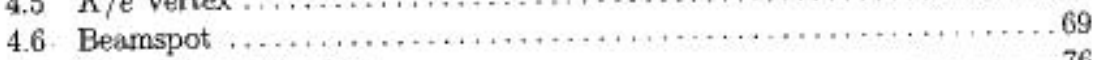

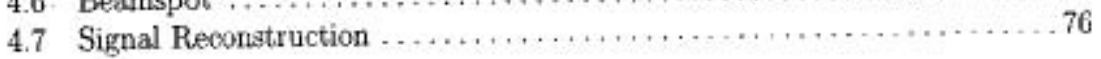

4.7 .1 Neural Network $D^{0}$ Momentum Estimator ................76

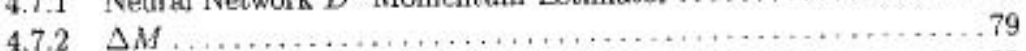

$4.7 .3 D^{0}$ Proper Lifetime .........................., 82

4.7 .4 Neural Network Event Selector . . . . . . . . . . . . . . . 88

4.7.4.1 Training Sample .......................... 88

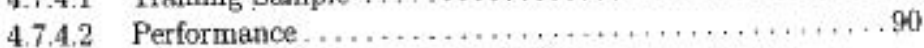

4.7 .5 Skim Event Selection . . . . . . . . . . . . . . . . . . . . . 92

4.7 .6 Final Event Selection ......................... 96

5. THE LIKELIHOOD FIT $\ldots \ldots \ldots \ldots \ldots \ldots \ldots \ldots \ldots$

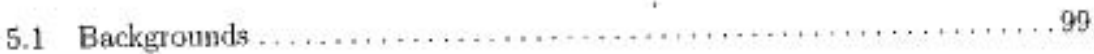

5.1 Combinatoric Background Estimation ................. 102

5.1 .2 Gamma Conversions ................................ 103

5.2 Analysis Method . . . . . . . . . . . . . . . . . . . . . . . . . . 103

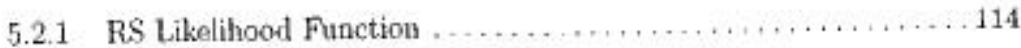

5.2.1.1 Unmixed Signal Events .................... 114

5.2.1.2 Random Combinatoric Backgrounds . . ........... 117

5.2 .1 .3 RS $D^{+}$Background ....................... 120

5.2.1.4 RS Global Likelihood Function................ 122 


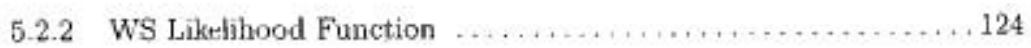

5.2 .2 .1 Mixed Sigmal Events ........................ 124

5.2.2.2 Random Combinatoric Backgrounds . ............ 128

5.2 .2 .3 Peaking $D^{+}$Background ...................... 129

5.2 .2 .4 WS Global Likelihood Function ................ 129

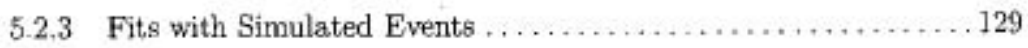

5.2 .3 .1 RS Unmixed Fit .......................... 131

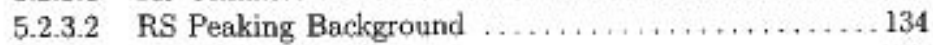

5.2 .3 .3 WS Mixed Fit . . . . . . . . . . . . . . . . . . . 139

5.2 .4 Fitting the Data ............................. 149

5.2 .4 .1 RS Data ................................ 149

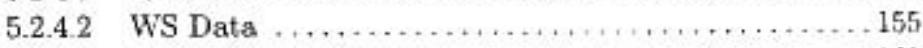

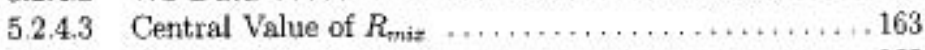

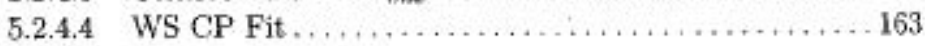

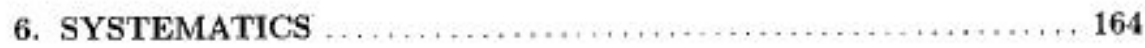

6.1 Cross-checks on the Final Result . . . . . . . . . . . . . . . . . . . . . . . 164

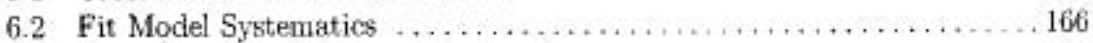

6.3 Reconstruction Efficiency Systematics ..................... 169

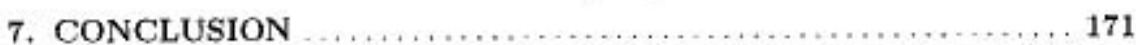

7.1 Extracting an Upper Limit ........................... 171

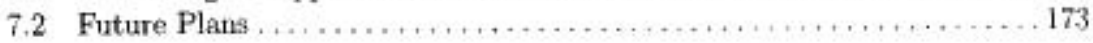

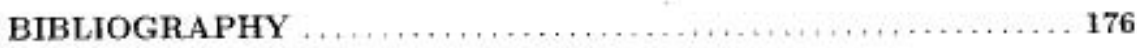




\section{LIST OF TABLES}

Table

Page

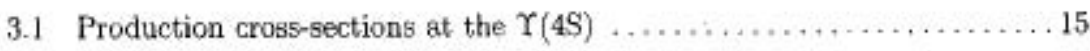

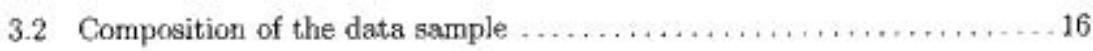

3.3 Composition of the simulated event sample. . . . . . . . . . . . . . 19

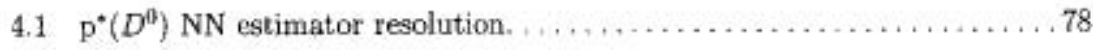

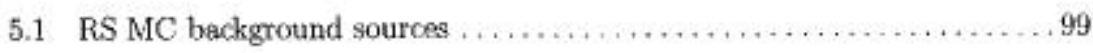

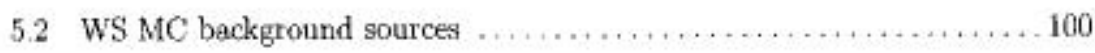

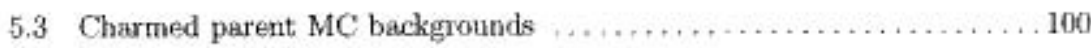

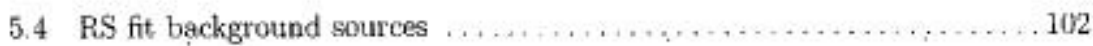

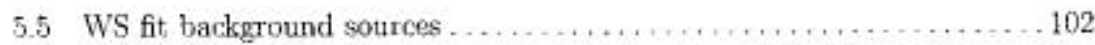

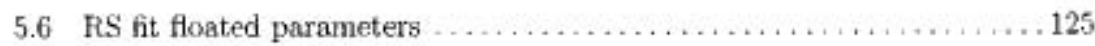

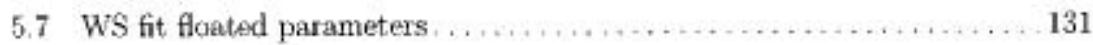

5.8 Mean and sigma of RS fit parameters taken from ensemble of MC

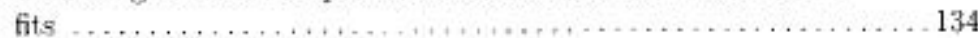

5.9 Mean and sigma of WS fit parameters taken from ensemble of MC

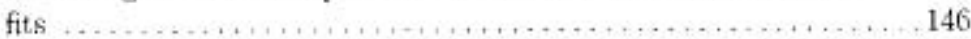

5.14 RS fit contral values and errots . . . . . . . . . . . . . . . . . . 150

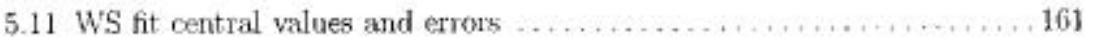

5.12 WS CP fit central values and errors $\ldots \ldots \ldots \ldots \ldots \ldots \ldots \ldots . \ldots \ldots . \ldots \ldots$

6.1 Non-systematic variations in the value of $\mathrm{R}(\mathrm{mix}) \ldots \ldots \ldots \ldots \ldots \ldots .165$ 
6.2 Systematic variations in the value of $\mathrm{R}(\mathrm{mix})$. 


\section{LIST OF FIGURES}

Figure Page

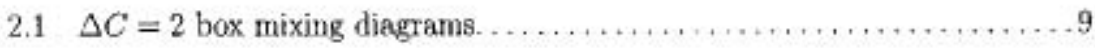

$2.2 \Delta C=2$ dipenguin mixing diagram. $\ldots \ldots \ldots \ldots \ldots \ldots \ldots \ldots, \ldots \ldots \ldots$

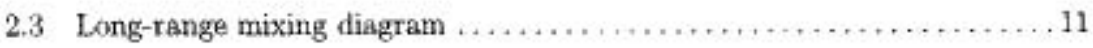

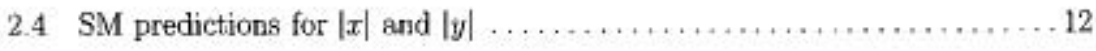

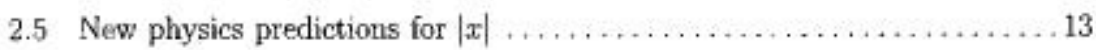

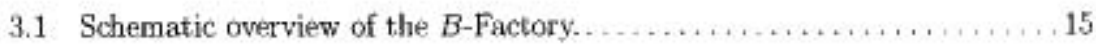

3.2 Daily recorded luminosity . . . . . . . . . . . . . . . . . . . 17

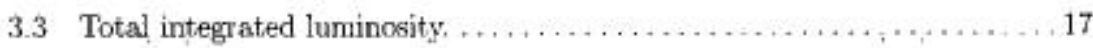

3.4 Babar detector in cut-away end view. . . . . . . . . . . . . 21

3.5 Bahar detector in longitudinal section. $\ldots \ldots \ldots \ldots \ldots \ldots \ldots \ldots, \ldots \ldots 22$

3.6 Schematic view of the SVT in fongitudinal section. $\ldots \ldots \ldots \ldots \ldots \ldots .23$

3.7 Schematic view of the SVT in transverse section. . . . . . . . . . . 24

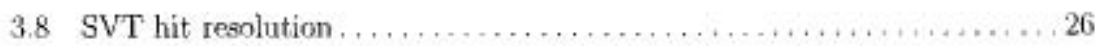

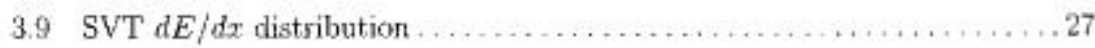

3.10 Schematic view of the DCH in longitudinal section $\ldots \ldots \ldots \ldots \ldots \ldots 28$

3.11 Schematic layout of $\mathrm{DCH}$ cteift cells... . . . . . . . . . . . . . . 29

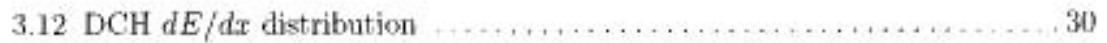

3.13 Histogram of DCH $d E / d x$ for kaons and pions $\ldots \ldots \ldots \ldots \ldots \ldots \ldots, 31$ 
3.14 DCH track reconstruction efficiency

3.15 SVT-only track reconstruction efficiency $\ldots \ldots \ldots \ldots \ldots \ldots \ldots, \ldots, 34$

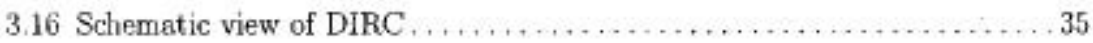

3.17 Schematic view of a DIRC quartz radiator bar $\ldots \ldots \ldots \ldots \ldots \ldots \ldots \ldots \ldots \ldots \ldots$

3.18 DIRC event display showing all hits in trigger window $\ldots \ldots \ldots \ldots \ldots . \ldots 39$

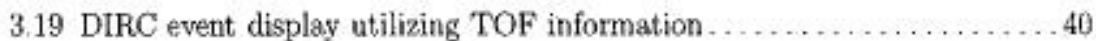

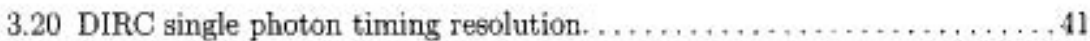

3.21 Difference between measured and expected Cherenkov angle $\ldots \ldots \ldots, \ldots 42$

3.22 Number of DIRC photons per track as a function of polar angle . . . . . 43

3.23 Charged kaon Cherenkov angle as \& function of momentum . . . . . . . .44

3.24 Charged pion Cherenkov angle as a function of momentum , ., ... . . . 45

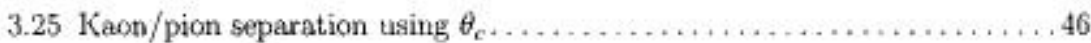

3.26 Kaon reconstruction efficiency and pion mis-identification rate ... . . . . 47

3.27 Longitudinal section of the EMC $\ldots \ldots \ldots \ldots \ldots \ldots \ldots \ldots \ldots \ldots, 48$

$3.28 E / p$ for electrons, pions and muons $\ldots \ldots \ldots \ldots \ldots \ldots \ldots \ldots \ldots, 50$

4.1 Kaon efficiency and misidentification rate $\ldots \ldots \ldots \ldots \ldots \ldots \ldots, 57$

4.2 Comparison of kaon efficiency and misidentification rates for varions identification schemes $\ldots \ldots \ldots \ldots \ldots \ldots \ldots \ldots \ldots . \ldots \ldots \ldots$

4.3 Representative distributions of EMC LAT for electrons...........6. 61

4.4 Representative distributions of EMC LAT for pions $\ldots \ldots \ldots \ldots \ldots \ldots, 62$

4.5 Representative distributions of EMC $\Delta \phi \ldots \ldots \ldots \ldots \ldots \ldots \ldots \ldots .63$

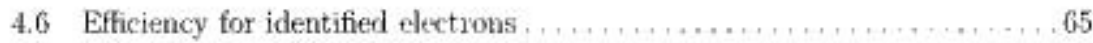

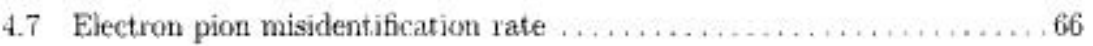




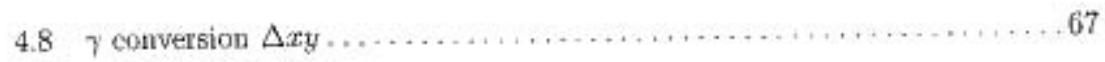

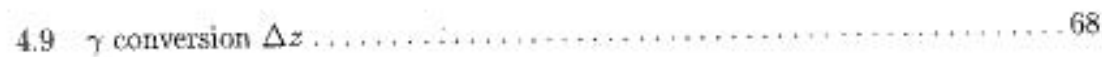

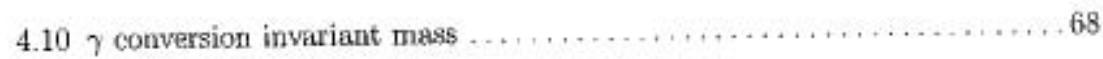

$4.11 \mathrm{RS} / \mathrm{WS}$ vertex pulls, .............................. 70

4.12 RS/WS TwoTrksVtx beamspot pults ................... 73

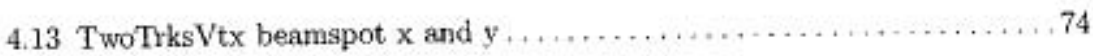

4.14 Decay length $\mathrm{v}$. run number $\ldots \ldots \ldots \ldots \ldots \ldots \ldots \ldots \ldots \ldots \ldots, 75$

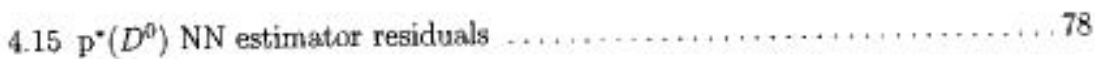

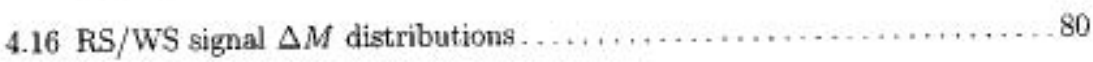

$4.17 \Delta M$ distributions for $\operatorname{RS} K$ and $K^{*}$ modes, ............... 81

$4.18 \mathrm{RS} / \mathrm{WS}$ reconstructed $D^{0}$ lifetime distribution $\ldots \ldots \ldots \ldots \ldots \ldots \ldots$

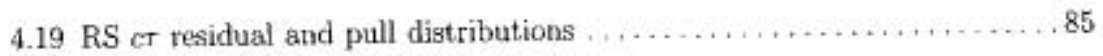

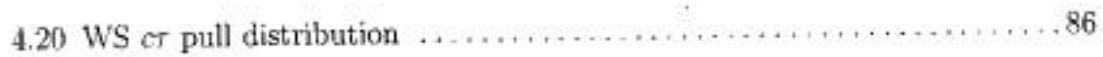

4.21 Mixed lifetime pull in bins of true lifetime $\ldots \ldots \ldots \ldots \ldots \ldots \ldots . . .67$

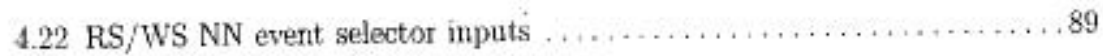

4.23 RS/WS NN selector output for simulated signal events ...........91

4.24 RS NN selector output for data and simulated events ............. 93

4.25 WS NN selector output for data and simulated events ............ 94

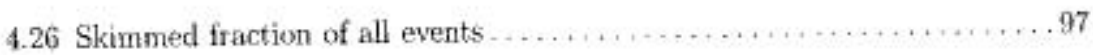

5.1 RS/WS random sombinatoric $\Delta M$ distributions $\ldots \ldots \ldots \ldots \ldots \ldots, 104$

$5.2 \Delta \mathrm{M}$ distributions for RS/WS events removed by gamma conversion

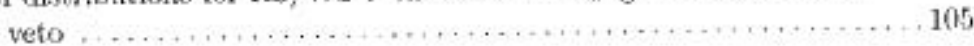

5:3 $\Delta M$ distributions for RS/WS data and simulated events $\ldots \ldots \ldots \ldots .107$ 
5.4 Normalized $\Delta M$ distributions for RS/WS data and simulated events . . . . . . . . . . . . . . . . . . . . . . . . . . . 108

5.5 or distributions for RS/WS data and simulated events . . . . . . . . . 109

5.6 Lifetime distributions for RS simulated and data events in $\Delta M$ signal

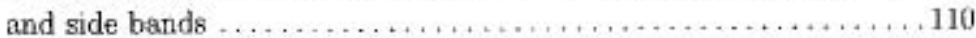

5.7 $\Delta M$ distributions for RS simulated and data events in lifetime signal and side bands ................................... 111

5.8 Lifetime distributions for WS simulated and data events in $\Delta M$

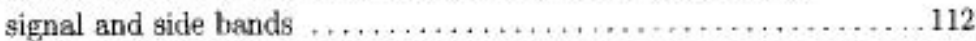

$5.9 \Delta M$ distributions for WS simulated and data events in lifetime signal

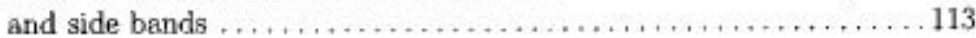

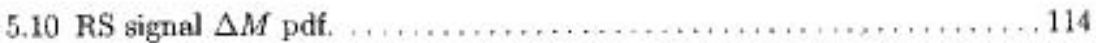

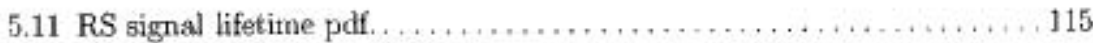

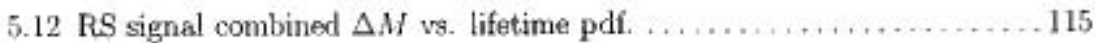

5.13 Full-range RS signal combined $\Delta M$ vs. lifetime pdf $\ldots \ldots \ldots \ldots \ldots \ldots$

$5.14 \mathrm{RS} / \mathrm{WS}$ random combinatoric $\Delta M$ pdf $\ldots \ldots \ldots \ldots \ldots \ldots \ldots \ldots \ldots \ldots \ldots$

$5.15 \mathrm{RS} / \mathrm{WS}$ representative zero lifetime pdf. $\ldots \ldots \ldots \ldots \ldots \ldots \ldots \ldots \ldots$

5.16 RS random combinatoric zero lifetime combined $\Delta M$ vs. lifetime pdf...................................., 119

5.17 Close-up of signal region for RS random combinatoric zero lifetime combined $\Delta M$ vs, lifetime pdf. . . . . . . . . . . . . . . . . 119

$5.18 \mathrm{RS} / \mathrm{WS}$ reduced $D^{0}$ lifetime pdf........................ 120

5.19 RS random conbinatoric reduced $D^{0}$ lifetime combined $\Delta M$ vs.

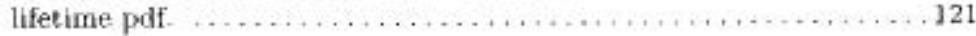

5.20 Close-up of signal region for RS random combinatoric reduced $D^{\mathrm{a}}$ lifetime combined $\Delta M$ vs. lifetime pdf. . . . . . . . . . . . . . 122

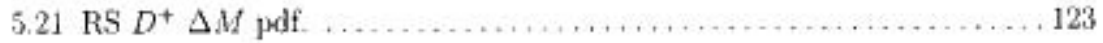


5.23 RS random combinatoric reduced $D^{+}$lifetime combined $\Delta M$ vs.

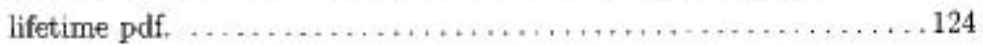

5.24 Close-up of signal region for RS random combinatoric reduced $D^{+}$

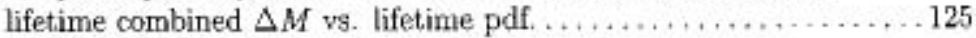

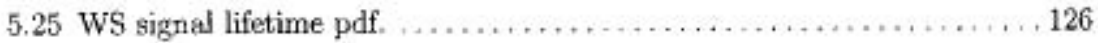

5.26 WS signal combined $\Delta M$ vs. lifetime pdf. . . . . . . . . . . . 127

5.27 WS signal combined $\Delta M$ vs, lifetime pdf displayed over the full combined backgroind + signal fit range. . . . . . . . . . . . . . . 127

5.28 WS random combinatoric reduced $D^{+}$lifetime combined $\Delta M$ vs.

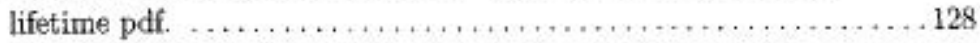

5.29 Close-up of signal region for WS random combinatoric reduced $D^{+}$

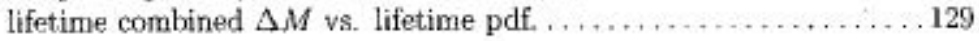

5.30 WS peaking reduced $D^{+}$lifetime combined $\Delta M$ vs. lifetime pdf. $\ldots, \ldots 130$

5.31 Close-up of signal region for WS peaking reduced $D^{+}$lifetime combined pdf . . . . . . . . . . . . . . . . . . . . . . . . . . 130

5.32 Projection in the PS $\Delta M$ sideband region of a fit to a simulated

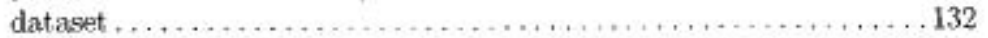

5.33 Projection in the RS $\Delta M$ signal region of a fit to a simulated dataget . . . . . . . . . . . . . . . . . . . . . . . . . . . . 132

5.34 Projection in the RS $c r$ sidebands of a fit to a simulated dataset . . . . 133

5.35 Projection in the RS cr signal region of a fit to a sirutated dataset .

5.36. Distribution of fit values from fits to the ensemble of RS simulated events datasets . . . . . . . . . . . . . . . . . . . . . . . . . . .

5.37 Distribution of fit values from fits to the ensemble of RS simulated

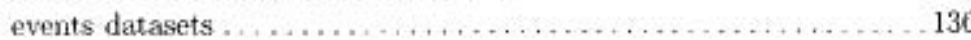

5.38 Distribution of fit values from fits to the ensemble of RS simulated events datasets . . . . . . . . . . . . . . . . . . . . . . . . . . . . 
5.39 Distribution of fit values from fits to the ensemble of RS simulated events datasets . . . . . . . . . . . . . . . . . . . . . . . 138

$5.40 \Delta M$ projection of a fit to a RS simulated dataset containing no unmixed signal events ........................., 139

$5.41 \mathrm{cr}$ projection of a fit to a RS simulated dataset containing no unrnixed signal events ............................. 140

5.42 Distribution of the fit number of RS peaking background events . . . . . 141

$5.43 \Delta M$ projection of a fit to a WS simulated dataset.............. 142

$5.44 \mathrm{cT}$ projection of a fit to a WS simulated dataset $\ldots \ldots \ldots \ldots \ldots \ldots . \ldots 143$

5.45 cr projection in the sideband region of a fit to a WS simulated

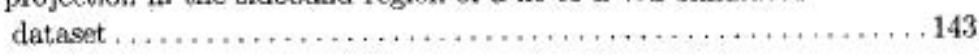

5.46 Distribution of fit values from fits to the ensemble of WS simulated events datasets . . . . . . . . . . . . . . . . . . . . . . 144

5.47 Distribution of fit values from fits to the ensemble of WS simulated events datasets . . . . . . . . . . . . . . . . . . . . . . . 145

5.48 Pull distributions for the fit value of $\mathrm{N}$ (mixed signal) $\ldots \ldots \ldots \ldots \ldots 147$

5.49 Mean of pull distribution for the fit value of $\mathrm{N}(\mathrm{mix})$ as a function of

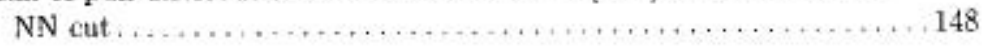

5.50 Projection in the $\Delta M$ signal region of a fit to the RS dataset ........151

5.51 Projection in the $\Delta M$ sideband of a fit to the RS dataset . . . . . . . 151

5.52 Projection in the $c r$ signal region of a fit to the RS dataset . . . . . . 152

5.53 Projection in the $c r$ sideband of a fit to the RS dataset $\ldots \ldots \ldots \ldots \ldots 152$

$5.54 \Delta M$ projections of a fit to the RS dataset in a ct region of high and low unmixed signal density . . . . . . . . . . . . . . . . . . . 153

$5.55 \mathrm{cr}$ projections of a fit to the RS dataset in a $\Delta M$ region of high and

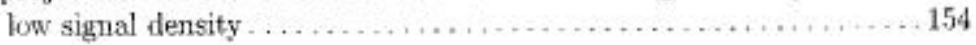

5.56 Distribution of NLL values from RS toy fits $\ldots \ldots \ldots \ldots \ldots \ldots \ldots, 156$ 
$5.57 \Delta M$ projection of a fit to the WS dataset $\ldots \ldots \ldots \ldots \ldots \ldots \ldots \ldots . \ldots \ldots 7$

$5.58 \mathrm{cr}$ projection of a fit to the WS dataset.................... 158

5.59 er projection of a fit to the WS dataset with a reduced vertical

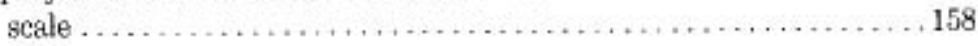

5.60 $\Delta M$ projections of a fit to the WS dataset in a cr region of high and

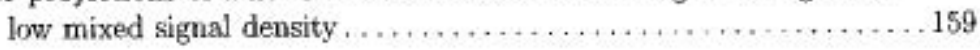

5.61 or projections of a fit to the WS dataset in a $\Delta M$ region of high

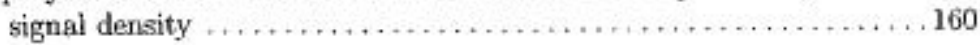

5.62 Distribution of NLL values from wS toy fits $\ldots \ldots \ldots \ldots \ldots \ldots \ldots \ldots \ldots$

6.1 Random combinatoric event mixing $\Delta M$ and $M(K e \pi)-M(K e)$ pdf

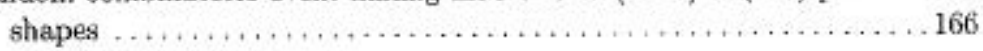

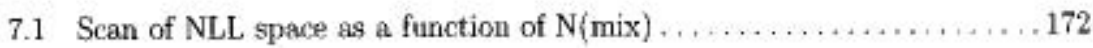

7.2 Charm mixing results in the $x, y$ mixing parameter plane $\ldots \ldots \ldots .174$ 